Review Article

\title{
Analyzing the Classification Techniques for Bulk of Cursive Languages Data: An Overview
}

\author{
Mu Hong $\mathbb{D}^{1,2}$ Shah Nazir $\mathbb{D}^{3},{ }^{3}$ Zhang Shuo, ${ }^{2,4}$ and Wang Guan ${ }^{2,4}$ \\ ${ }^{1}$ Hohai University School of Public Administration, Nanjing 210000, China \\ ${ }^{2}$ Sangmyung University the Graduate School, Seoul 03016, Republic of Korea \\ ${ }^{3}$ Department of Computer Science, University of Swabi, Swabi, Pakistan \\ ${ }^{4}$ Nanhang Jincheng College School of Art and Communication, Nanjing 210016, China \\ Correspondence should be addressed to Mu Hong; clarke@hhu.edu.cn and Shah Nazir; snshahnzr@gmail.com
}

Received 28 December 2020; Revised 26 January 2021; Accepted 8 February 2021; Published 23 February 2021

Academic Editor: Muhammad Arif Shah

Copyright $\odot 2021 \mathrm{Mu}$ Hong et al. This is an open access article distributed under the Creative Commons Attribution License, which permits unrestricted use, distribution, and reproduction in any medium, provided the original work is properly cited.

\begin{abstract}
The remarkable growth of texts both in online and offline is becoming a challenging issue which need exploration for further research. Diversities of regional and cultural changes have produced diverse languages as a source of communication. Variations of styles are existing for handwritten texts which is due to varying writing styles. The research area of text recognition is matured which has increased a number of directions in the area of research. A detail report of the existing literature is needed which can help practitioners and researchers to use the existing evidence and provide new solutions for identification of cursive languages and to optimize the ability of recognition for cursive text. For facilitating the researchers and practitioners by providing in-depth analysis of the existing literature, the proposed study provide a detail report through which researchers can get benefit of the literature and devise new solutions. This study is based on searching various popular libraries for identifying relevant materials associated with the proposed study.
\end{abstract}

\section{Introduction}

With the passage of time, a significant growth of texts arises both in online and offline. This growth is becoming a challenging issue for researchers which need consideration for further research. Different diversities exist in the form of regional and cultural changes which have produced various languages for communication. The growth of computing devices and facilitations of low-cost access to Internet has presented new directions of retrieving information from digital libraries [1]. In accumulation to this, image, audio, and video archive, the collection of documents be significant part of digital record. For the last couple of years, several organizations have digitized their document collections and have made them available online to facilitate retrieval and for community use. The issue of these documents is that they are mostly in image format which are neither editable and nor searchable. Such documents can occupy more storage space as compared to the textual format. Also, if such images are accessed/processed through the Internet, it will require more bandwidth. Keeping in view this issue, it was desired by the researchers to convert such images into textual format which can then be easily accessed and processed.

Optical character recognition (OCR) system can facilitate the conversion of document image into textual format. With the advancements, the OCR system for many scripts/ languages is in the early stage of research. Such languages include Urdu, Pashto, Persian, Arabic, and many others. With the help of handwriting recognition (HWR), the written text can be transformed into a symbolic depiction. This transformation can facilitate the interaction of human and computer applications like mail sorting, cheque verification, image recognition, office automation, and interaction of human computer [2-4]. The language recognition of handwriting recognition of Latin and Chinese has been researched and has achieved significant success. Parallel to this, the research in other languages like HWR of Urdu, Arabic, Persian, and Pashto is less. The reason is that there 
are more variations of writing styles and complexity. The handwriting recognition can be categorized into offline and online systems. The offline text recognition is difficult to recognize due to the reasons that these are available in the form of images with written text, while the online recognition of text is easy; as in such system, there is no need of sequence or order of writing [5].

The field of text recognition is matured which has amplified the directions in the area of research. Detail of the existing literature is desirable which can assist practitioners and researchers to use the existing evidences and provide new solutions for identification of cursive languages and to optimize the ability of recognition for cursive text. For facilitating the researchers and practitioners by providing indepth analysis of the existing literature, the proposed study provide a detail report through which researchers can get benefit of the literature and devise new solutions.

The organization of the paper is as follows: Section 2 shows the related work to the cursive script and language recognition, and the approaches, techniques, and methods used are described. Section 3 shows the analysis of the existing work associated with the text recognition. The paper is concluded in Section 4.

\section{Approaches for Cursive Script Recognition}

Researchers are trying to devise algorithms, novel approaches, and solutions for the recognition of cursive script and languages. Shaikh and Shaikh [6] proposed an algorithm of parallel thinning for cursive or noncursive languages by defining a customized set of preservation rules through pixel arrangement grid template, producing strong restriction to noise and speed. The results of experiments showed significant achievements over the other cursive languages such as Sindhi, Urdu, and Arabic and noncursive languages such as Chinese, English, and numerals. Dhande and Kharat [7] presented an approach for cursive language recognition of handwriting in English language. Mostly, in the cursive handwriting of English script, the word characters are connected to each other. So the feature extraction and segmentation of English cursive script is difficult. The approach has used the method of horizontal and vertical projection for segmentation. The algorithm of convex hull is used for extracting features, and support vector machine is used as algorithm for recognition and classification. Chinese language is considered to be a widely used language around the globe. The script of Chinese is the most distinctive traditional culture and calligraphic art of China. Research is needed for its connecting writing recognition for text on cursive images. Qin et al. [8] offered a method for cursive text detection for the dataset and is known as SE-seglink. The feature extraction from image is enhanced through this method. The authors designed a dataset containing 523 images for Chinese cursive text. Comparing to the available approaches, the offered approach is performing better in terms of recognizing the cursive images. The effectiveness of the approach is tested through performing comparative experiments.

Ueki et al. [9] proposed an approach for recognition of consecutive Kuzushiji characters through multiple candidate regions as input to a neural network. An assessment through database of images of three consecutive Kuzushiji characters confirmed that the approach proposed is having greater rate of accuracy compared to the approach in which the character of images were cropped according to the boundary detected. Han and Sethi [10] proposed a method which uses heuristic rule set for determining probable boundaries of letter in the image with word curved. The heuristic rules are based on the relations existing between assured topologic and geometric features and the character of English language. A system of segmentation has been built which integrates the proposed system for performing segmentation on postal address images. Various steps are involved in the preprocessing of extracting handwritten words from postal envelope and the step of normalization for allowing variation in thickness of pen and witting tilt. The results obtained from the experiments revealed that the approach is efficient and able for locating the boundaries of letter in cursive words accurately. Kim and Lee [11] offered an approach of unified network for recognition of handwritten text in different languages. The system can be used for any grouping of phonetic writing systems such as Japanese, Arabic, and Tai.

Sternby and Friberg [12] presented an approach for interaction of dictionary in recognition of online cursive script. With the help of segmentation graph, all the probable paths are retrieved for corresponding to words in a dictionary in an effective way. The study also deals with the treating of secondary strokes in online segmentation graph. The approach was tested with huge data with good results. Ahmad et al. [13] proposed an approach for finding out the alternate recognizable unit in the cursive script of Pashto. The alternatives are primary ligature and ligature. A corpus of 2313736 words of Pashto is extracted from different sources of web, and 19268 unique ligatures were identified in the cursive script of Pashto. The results showed that 7000 ligatures showed $91 \%$ portion of corpus of Pashto words, and 7681 primary ligatures were identified representing the shapes of all the ligatures. Hassan [14] proposed a system for recognition of cursive Arabic writing. The issues arise due to the personal attitude, style variations, and various levels of writing. The system is based on recognition of hierarchal strategy. With the incorporation of grammar and parser, a system of linguistic recognition was developed. Hashemi et al. [15] designed a recognition system for the Persian text. The system contains a stage of segmentation to separate the character that is constituent. This stage is beneficial for italic or highly declined Latin text. The study presented a segmentation algorithm with two steps. In the first step, nonoverlapped and separate isolated characters are separated, while the second step segments nonassociated characters that are overlapped. The approach was tested on the script of real world and showed an accuracy of $99.7 \%$.

\section{Analyzing the Existing Studies for Cursive Script/Languages}

The following subsections present the related work and analysis of the cursive script/languages. 
3.1. Existing Research for Supporting Recognition of Cursive Script. Recognition of cursive languages in effective way is become challenging issue for researchers and practitioners. Diverse approaches have been proposed to tackle the issue of cursive languages from different perspectives. Erdogan and Ozge [16] suggested a study for analyzing the cursive handwriting of likely primary school teachers from the legibility viewpoint. The nature of the study is perspective with the aim to portray the available state of affairs, making use of qualitative methods. The study consist of 130 potential primary school teachers and were asked for copying the text presented by researchers using cursive handwriting. The cursive handwriting was examined through "cursive handwriting basement form." The study showed that the handwriting of teachers was sufficiently legible. Samanta et al. [17] proposed a hidden Markov model-based online unconstrained word recognition of handwritten samples. The system involved the key steps which are the handwriting segmentation into substrokes, extracting features from substrokes and recognition. For the task of segmentation, a strategy of discrete curve evolution is proposed. Then various linear and angular features are extracted from substrokes of samples of word and are modelled as feature vectors produced from a mixture distribution. The algorithm of Baum-Welch parameter estimation was used for handling the spherical linear correlated data for constructing the hidden Markov model. At last, the recognition classifier was designed for handwritten word samples. The results demonstrated that Bangla and Latin scripts have good performance of the suggested scheme of recognition. Camastra [18] proposed a recognizer approach for cursive characters which is a module in the recognition of any cursive word based on the approach of segmentation and recognition. With the help of support vector machine and neural gas, the classification of character is achieved. For verification of lower and upper case version of various letters, the neural gas was used, while for recognition of character, the SVM was used. The dataset of 57293 characters was considered for training and testing of the recognizer for cursive characters. The results reveal good performance by the use of SVM and showed better efficiency.

Darwish and ELgohary [19] presented an approach of bio-inspired expert system for printer forensics that incorporate both the features of texture and niching genetic search for selecting effective sufficient minimized feature set. The approach k-nearest neighbours was used for differentiating the printer brand for its simplicity. The results reveal that the approach is having high accuracy of classification and can take less time. Wen et al. [20] proposed a model of hierarchal deformation for describing the online cursive Chinese character deformation. The approach includes two levels: firstly, matching two sequences of turn points which are extracted from the reference and input characters for describing the matches of stroke. Then, the constrained parabola transformation is used for reducing the difference between the matched strokes correctly. The results show that hierarchal deformation approach is effective to the deformation of cursive Chinese character with less computational cost. Lee and Verma [21] proposed a new binary segmentation algorithm for reducing the issue of chain failure risk in the course of validation and improved the accuracy of segmentation. The binary segmentation algorithm is a combination segmentation approach including validation and over-segmentation. The validity of the research was carried out on benchmark of CEDAR database, and the results showed better performance. EL-SHEIKH and GUINDI [22] designed a recognition system for Arabic text. The approach contains segmentation stage for recognition of Arabic cursive words that are typewritten. The system showed 99\% recognition rate. Bhunia et al. [23] presented an approach of cross language platform for recognition and spotting of handwritten word. The approach is presented for scripts of low resource where training is done with huge dataset of an accessible script and test is done on the other script. The approach was tested on three Indic scripts including Devanagari, Bangla, and Gurumukhi.

Chandio et al. [24] presented a dataset for the detection of Urdu text, and recognition in natural scene images is analyzed. Above 2500 natural scene images were collected for developing dataset through the digital camera and with a mobile camera. Three datasets were developed including cropped word images, isolated Urdu character images, and end-to-end text spotting. The emphasis was given to the Urdu text instances. The approach can be used for performing detection and recognition of Urdu text as well as end-to-end recognition in natural scenes. Development of these datasets can provide help in developing Persian and Arabic natural scene text recognition and detection. Aisyah et al. [25] carried out a research for designing and developing learning materials for students for learning Japanese language as foreign language at the Universiti Kebangsaan Malaysia. The research carried out a survey of commercially produced text book and preproduction stage focusing on needs analysis from students. Abuhaiba [26] presented an approach for identification of cursive language or discrete script enclosed in an image document. The approach is based on extraction of set of global templates shared between languages and scripts with common shapes of symbols. It saves time of processing and requirement of memory in the execution of program. The approach performed one-dimensional normalization like retaining width-to-height ratio. The authors recommended the approaches that have good accuracy and speed for commercial use of OCR products Table 1 shows some of the approaches/methods used for recognition of cursive script/ languages from different perspectives.

\subsection{Analyzing the Literature for Cursive Script Recognition.} Recognition of cursive script/language is considered to be important for different purposes. This recognition of cursive script from the images can save time and storage memory. As these are in textual format, different approaches have been proposed. AlKhateeb et al. [37] have used the hidden Markov models for recognition of word-based offline text. Three stages are involved in the method including preprocessing, feature extraction, and classification. Initially, the words from the scripts of input are segmented and normalized, then features are extracted from the segmented 
TABLE 1: Approaches used for cursive text/script/language recognition.

\begin{tabular}{|c|c|c|c|c|}
\hline Reference & Authors & Method & Year & Article type \\
\hline [27] & J. B. Hellige and M. M. Adamson & Hemispheric differences in processing handwritten cursive & 2007 & Journal \\
\hline [28] & A. Jalali and M. Lee & $\begin{array}{l}\text { High cursive traditional Asian character recognition using integrated } \\
\text { adaptive constraints in ensemble of DenseNet and inception models }\end{array}$ & 2020 & Journal \\
\hline [29] & W. Cho, S.-W. Lee, and J. H. Kim & $\begin{array}{c}\text { Modeling and recognition of cursive words with hidden Markov } \\
\text { models }\end{array}$ & 1995 & Journal \\
\hline [30] & S. Naz et al. & $\begin{array}{c}\text { Offline cursive Urdu-Nastaliq script recognition using } \\
\text { multidimensional recurrent neural networks }\end{array}$ & 2016 & Journal \\
\hline [31] & M. Schambach & Recurrent HMMs and cursive handwriting recognition graphs & 2009 & Conference \\
\hline [32] & T. G. Rose and L. J. Evett & Semantic analysis for large vocabulary cursive script recognition & 1993 & Conference \\
\hline [33] & $\begin{array}{l}\text { R. J. Kannan, R. Prabhakar, and } \\
\text { R. M. Suresh }\end{array}$ & Off-line cursive handwritten Tamil character recognition & 2008 & Conference \\
\hline [34] & $\begin{array}{l}\text { A. A. Chandio, M. Asikuzzaman, } \\
\text { and M. R. Pickering }\end{array}$ & $\begin{array}{c}\text { Cursive character recognition in natural scene images using a } \\
\text { multilevel convolutional neural network fusion }\end{array}$ & 2020 & Journal \\
\hline [35] & $\begin{array}{l}\text { J. Danna, D. Massendari, } \\
\text { B. Furnari, and S. Ducrot }\end{array}$ & $\begin{array}{l}\text { The optimal viewing position effect in printed versus cursive words: } \\
\text { Evidence of a reading cost for the cursive font }\end{array}$ & 2018 & Journal \\
\hline [36] & B. Verma and H. Lee & $\begin{array}{l}\text { Segment confidence-based binary segmentation (SCBS) for cursive } \\
\text { handwritten words }\end{array}$ & 2011 & Journal \\
\hline
\end{tabular}

words, and then these features are integrated for classification purpose. The database of IFN/ENIT was used which contains 32492 words of Arabic handwritten notes. The approach delivered better performance compared to the existing approaches. Abu-Ain et al. [38] proposed a detection approach for baseline and straightness for the text of cursive handwritten notes. The approach is based on the analysis and extraction of directions features from subwords of the text skeleton. The text of Arabic language was considered as a case study. The results revealed that the approach is efficiently working and tested on Arabic dataset. Mouhcinea et al. [39] proposed a method of Arabic handwritten cursive text based on the hidden Markov model. The experimental results of the images of IFN/ENIT database benchmark revealed that the suggested approach enhanced recognition. Manjusha et al. [40] proposed an approach which aimed for building the databases of handwritten character image for the script of Malayalam language. The samples of handwritten collected from 77 native Malayalam writers. The contour model-based image segmentation algorithm was used for extracting the character images from the data sheets of handwritten. Features extraction techniques were used for extracting features. The scattering convolution network-based feature descriptors achieved a recognition accuracy of $91.05 \%$ which is the highest among the available feature descriptors. Naz et al. [41] reviewed the literature on OCR associated with the Urdu cursive scripts. The Pashto, Urdu, and Sindhi languages are described with focus on the script of Nasta'liq and Naskh.

Apart from the above literature, various popular libraries such as ScienceDirect, IEEE, Springer, and Wiley Online were used to search for achieving the most relevant materials. These libraries were only considered due to the reasons that these are only publishing peer reviewed and quality research. Figure 1 shows the initial results of the search process in the mentioned libraries. The figure shows that more materials were obtained in the library of Springer followed by the ScienceDirect.

Initially the library of ScienceDirect was searched, and the results were depicted in figures. Figure 2 shows the publication titles with the number of articles.

Figure 3 represents the type of articles in the given library.

Figure 4 represents the number of articles in the given years. Figure 5 shows the subject areas in the given libraries.

The library of IEEE was searched and the topics of articles are shown in Figure 6.

The article type is shown in Figure 7 where most of the papers are published as conference papers.

The locations where the conferences held were identified in the study conducted. Figure 8 depicts the locations of conferences held.

After this, the library of Springer was searched for identification of relevant materials and their analysis. Figure 9 represents the various disciplines of articles. The figure shows that more articles were published in the area of Computer Science.

Figure 10 shows the subdisciplines of the areas with total of publications.

The article type was identified in the given library. Figure 11 shows the total number of publications based on the article types. 


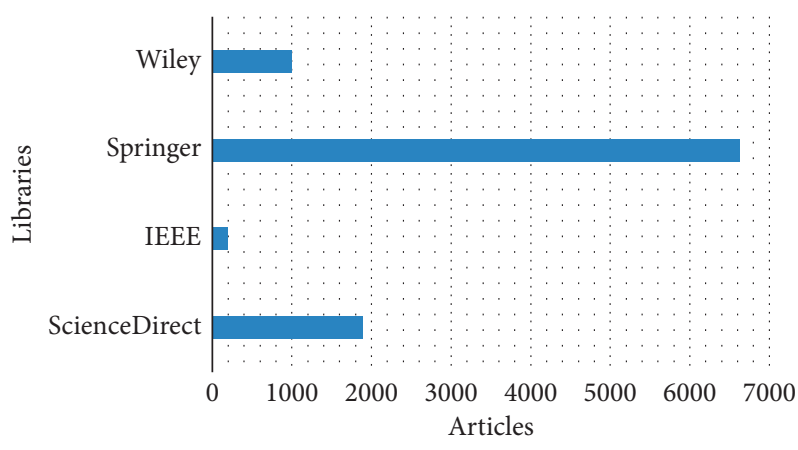

FIgURE 1: Search process of libraries.

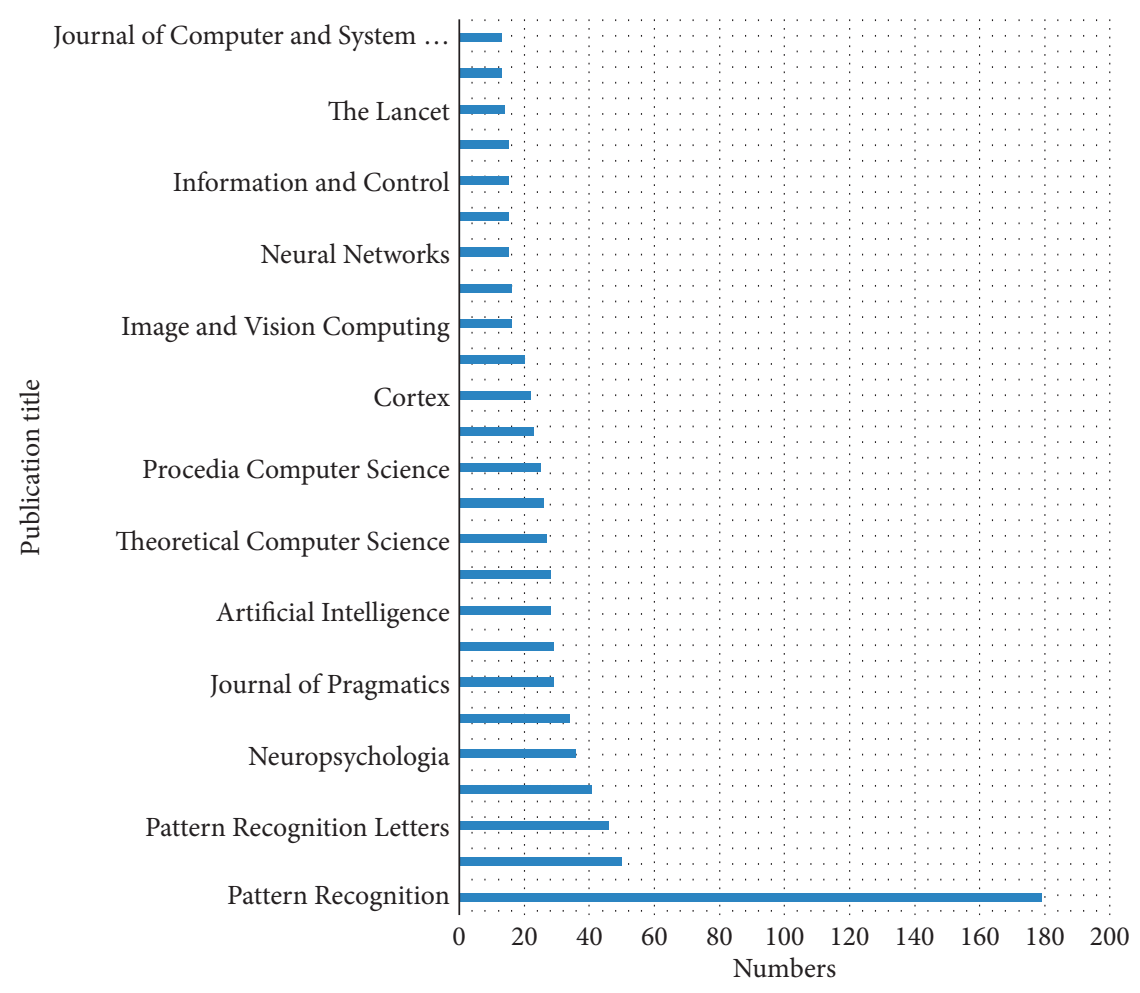

Figure 2: Publication title along with the papers.

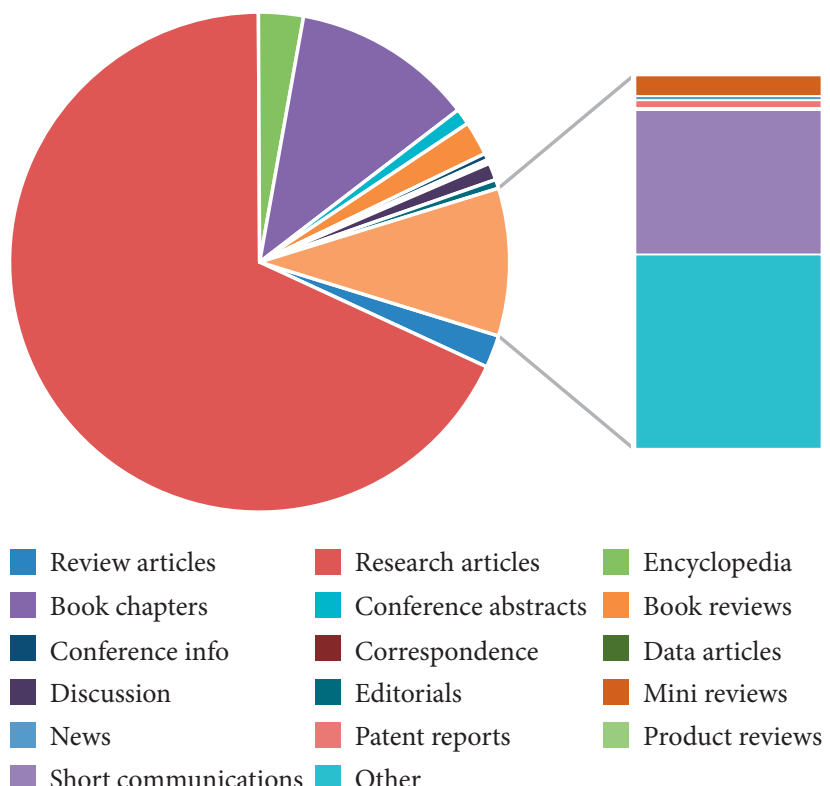

Figure 3: Type of articles. 


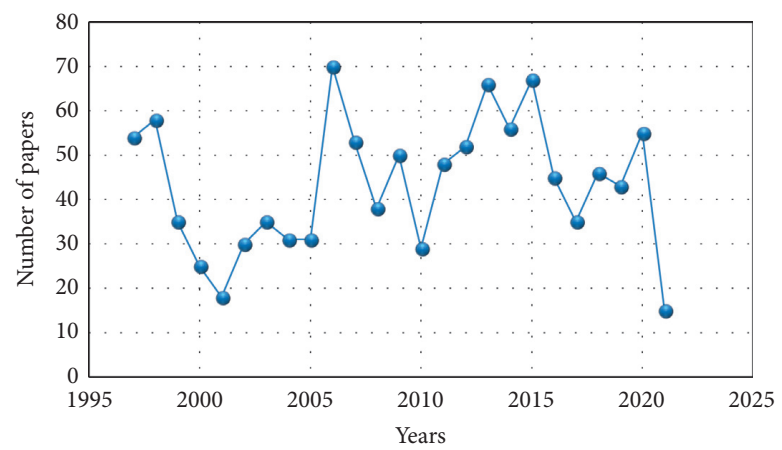

Figure 4: Number of publications in the given years.

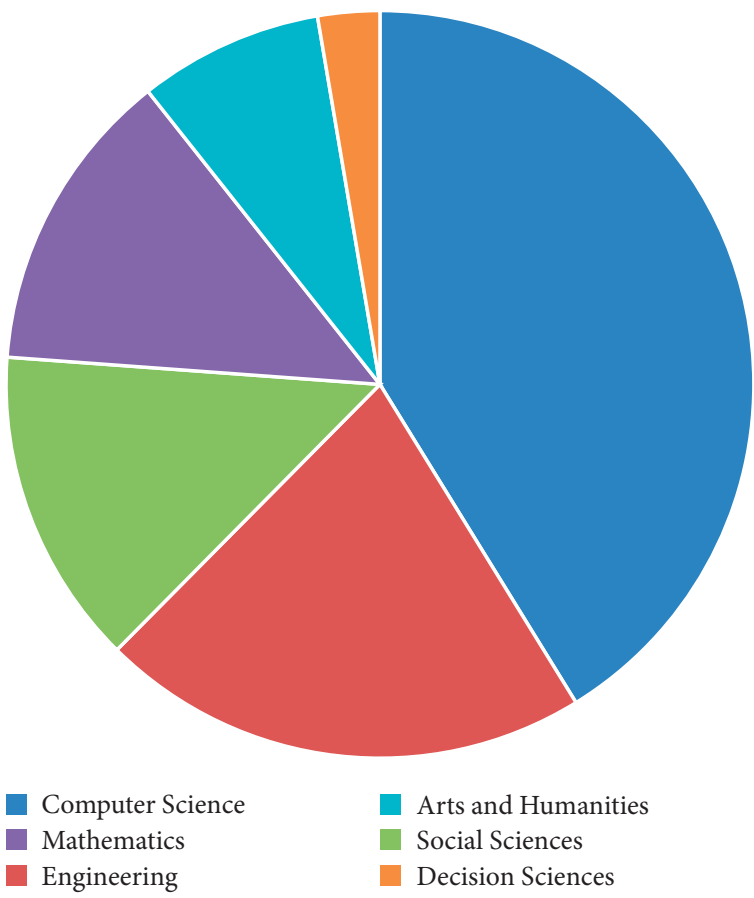

FIGURE 5: Subject areas.

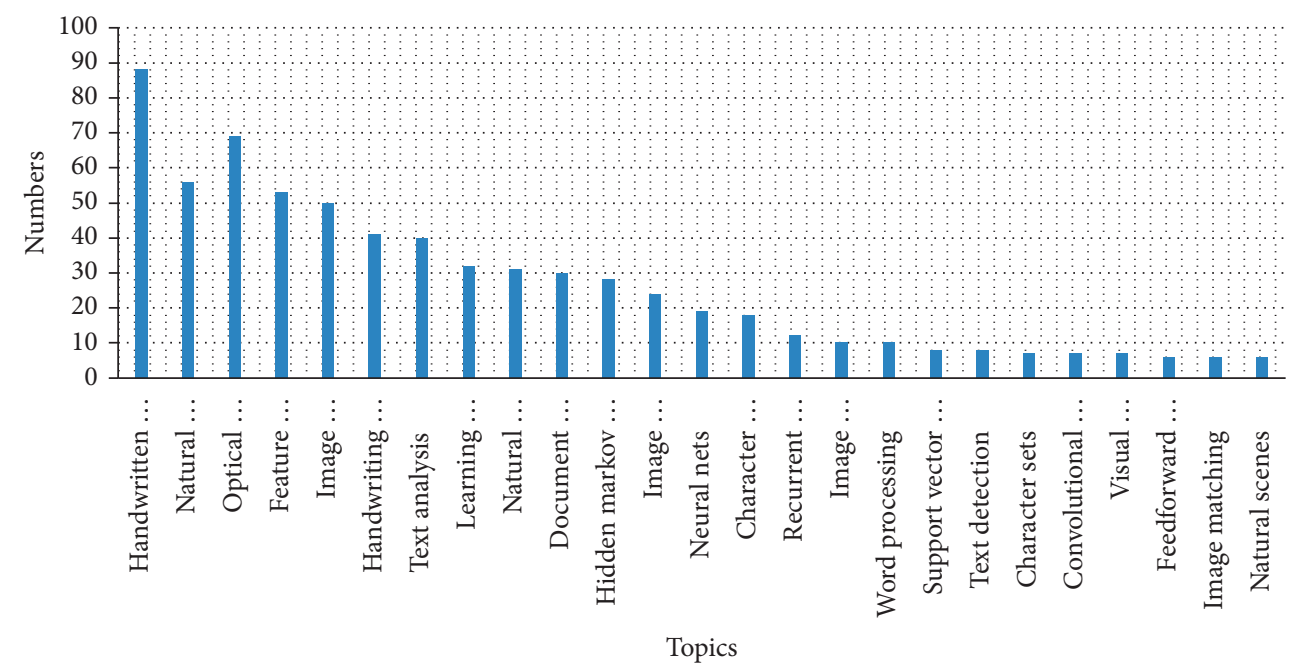

Figure 6: Topics of publications. 


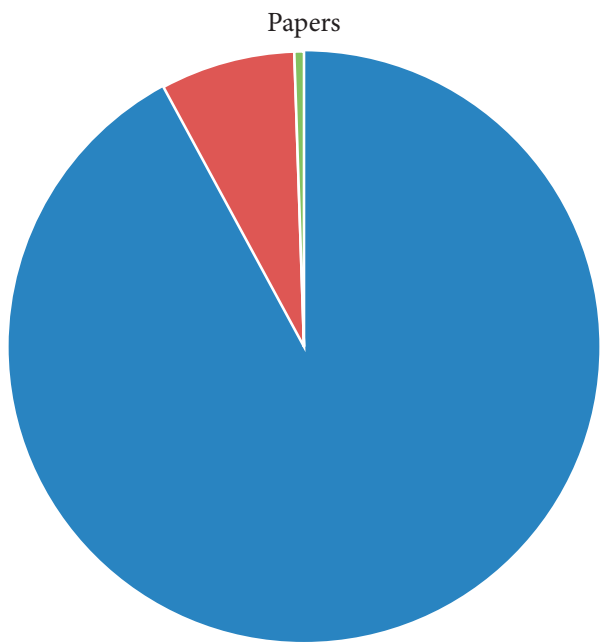

Conferences

Journals

Magazines

Figure 7: Publication types.

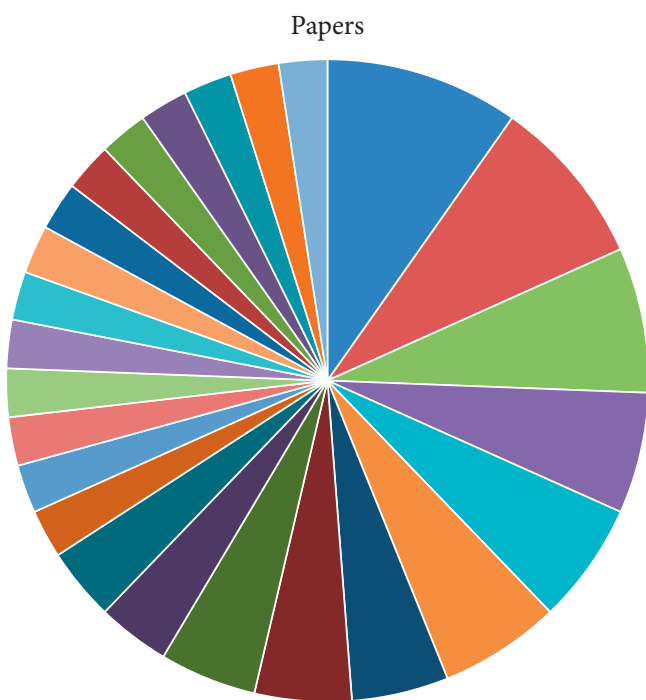
Barcelona
Bari
London, UK
Niagara Falls, NY
Beijing, China
Chennai
Heraklion
London

Islamabad

Sydney, Australia

Montreal, Quebec, Canada

- Parana

Tunis

Cambridge

Edinburgh, UK

Ulm, Germany

Kuala Lumpur

Montreal, Que.

- Shenzhen

Agadir

Chengdu

Gold Cost, QLD

FIgURE 8: Location of conferences held. 


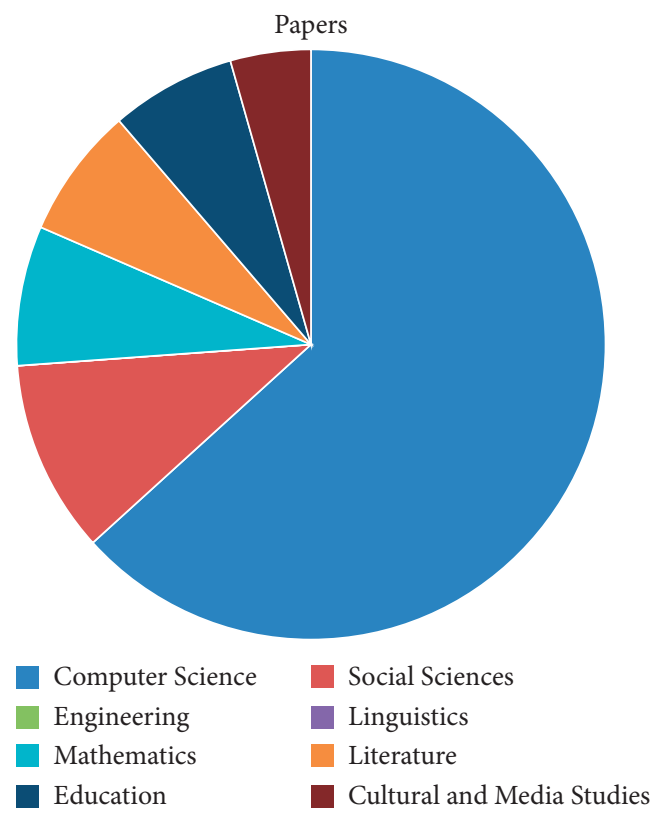

FIgURE 9: Discipline of publications.

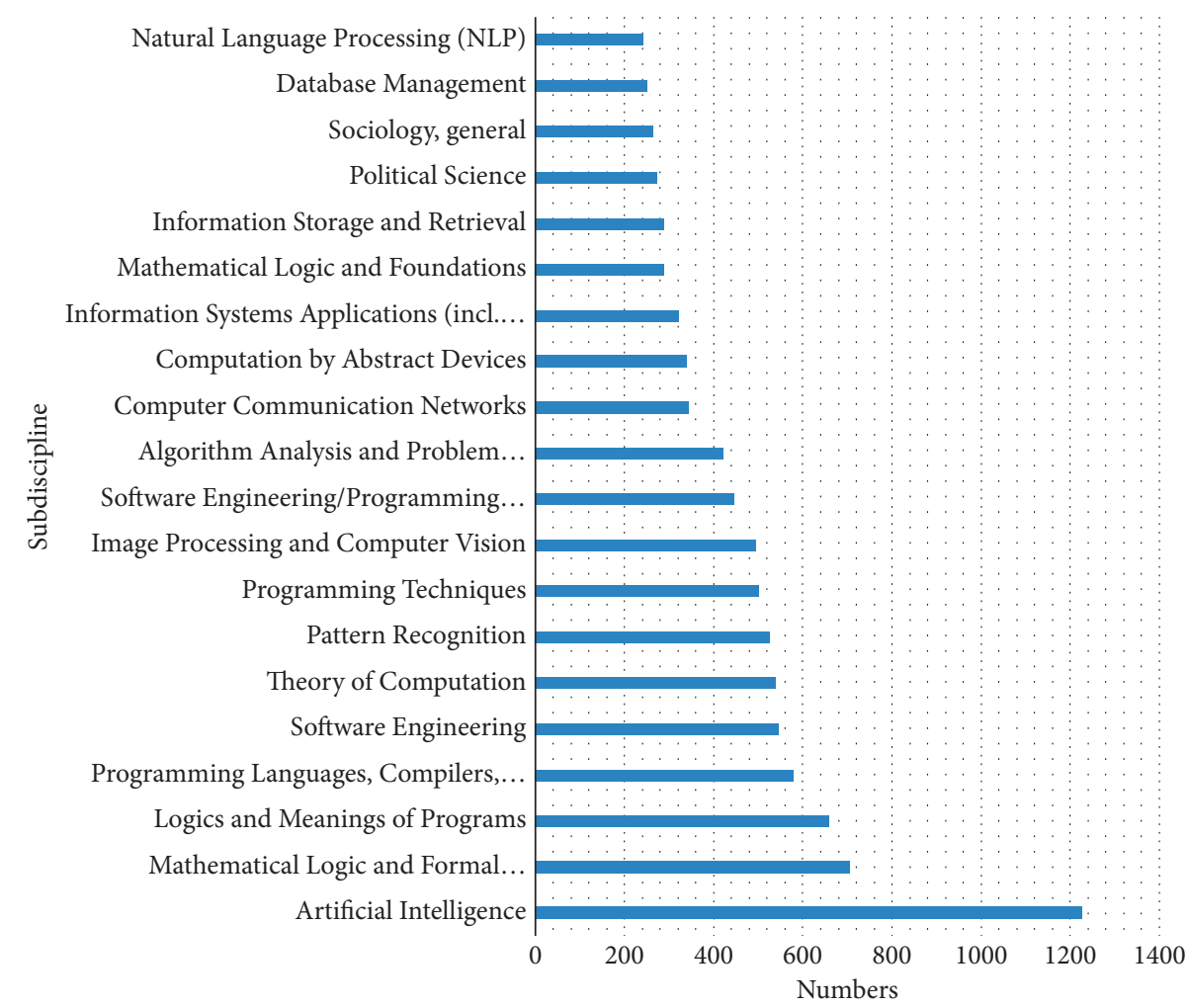

Figure 10: Subdisciplines of the areas with publications. 


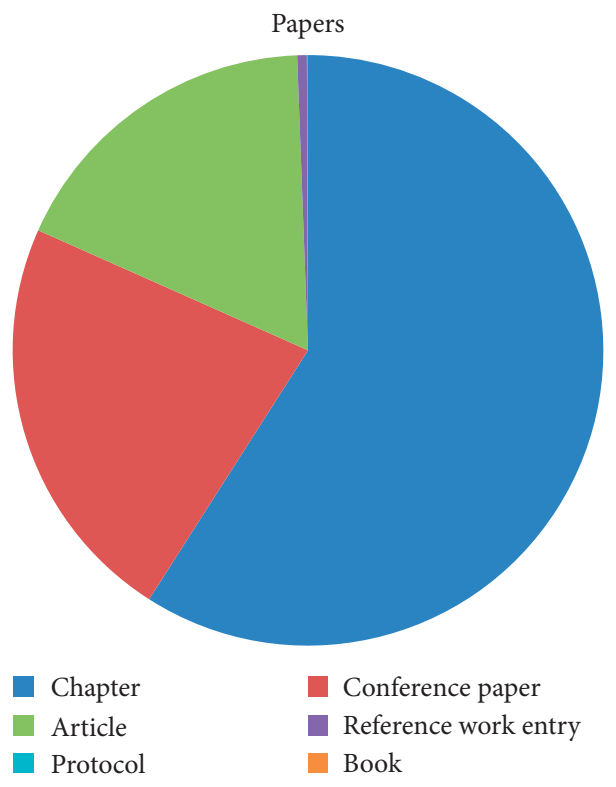

Figure 11: Article types with publications.

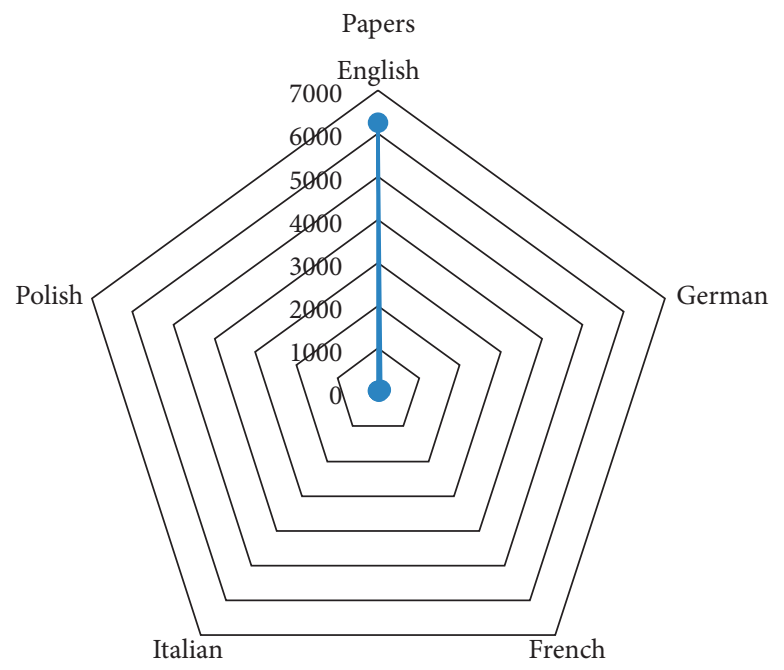

FIGURE 12: Languages of publications.

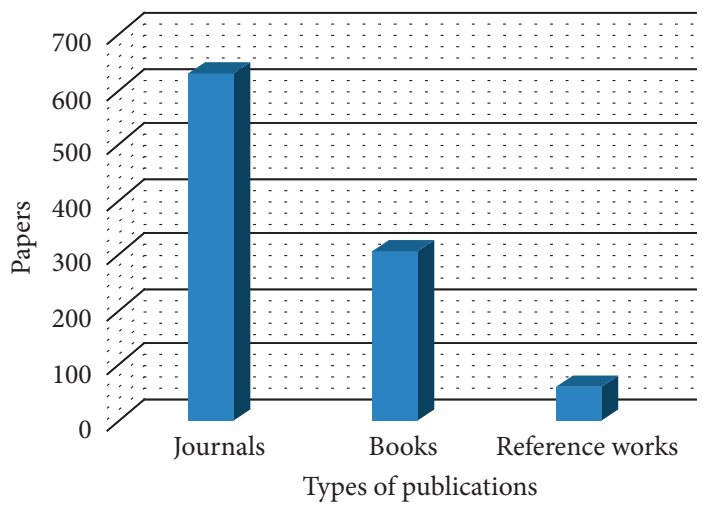

Figure 13: Types of publications with total numbers. 


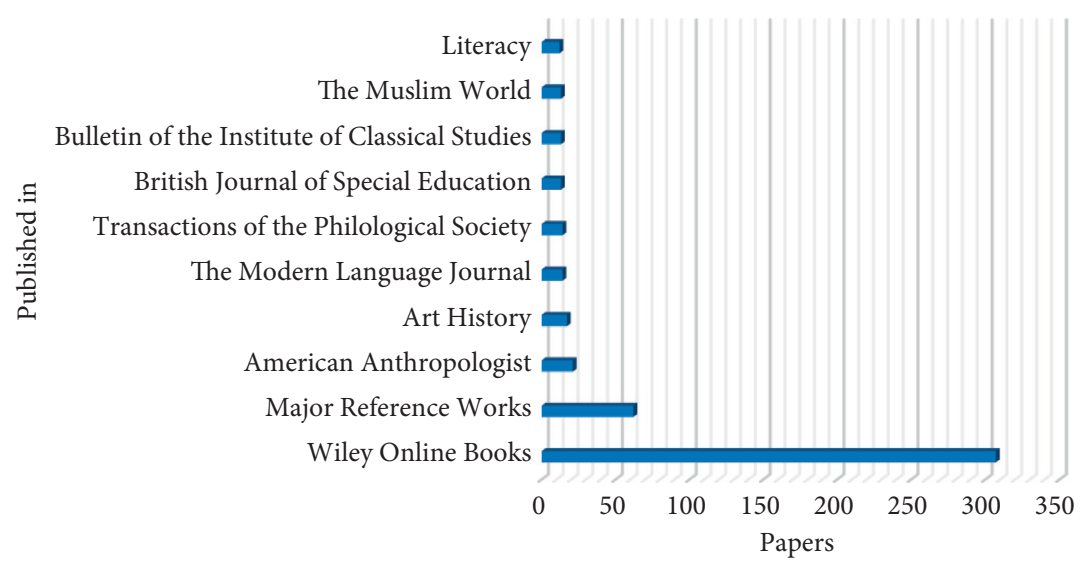

Figure 14: Papers published in with the number of articles.

The study also focused on the language of publications. Figure 12 shows the language of articles with the total number.

Figure 13 depicts the type of publications in the Wiley online library.

Figure 14 represents the papers published in with the number of articles.

\section{Conclusion}

With the recent advancements of modern technology and innovation in the field of machine translation, a significant growth of texts arises both in online and offline scripts. This growth is becoming a challenging issue for researchers which need consideration for further research and exploration. Diversities exist in the form of regional and cultural changes. The diversities of regional and cultural changes have produced diverse languages as a source of communication. Variations of styles exist for handwritten texts which are due to varying writing styles. The research area of text recognition is matured which has increased the directions in the area of research for exploration. A detail report of the existing literature is necessary which can support practitioners and researchers to use the existing literature as evidence and provide new solutions for identification of cursive languages and to optimize the ability of recognition for cursive text. The current study has provided a detail report through which researchers can get benefit of the literature and devise new solutions. The study is further facilitating the researchers and practitioners by providing in-depth analysis of the existing literature.

\section{Data Availability}

No data are available.

\section{Conflicts of Interest}

The authors declare no conflicts of interest.

\section{References}

[1] A. Bhardwaj, A. Thomas, Y. Fu, and V. Govindaraju, "Retrieving hand writing styles: a content based approach to hand written document retrieval," in Proceedings of the 2nd International Conference on Frontiers in Handwriting Recognition (ICFHR10), pp. 265-270, Kolkata, India, November 2010.

[2] S. Alma'Adeed, C. Higgins, and D. Elliman, "Off-line recognition of handwritten Arabic words using multiple hidden Markov models," Knowledge Based Systems, vol. 17, pp. 75-79, 2004.

[3] Y. Kessentini, T. Paquet, and A. M. Benhamadou, "Multiscript handwriting recognition with n-streams low level features," in Proceedings of the 19th Internet. Conf. Pattern Recognition (ICPR), Tampa, FL, USA, December 2008.

[4] M. R. Al-Hajj, L. Likforman-Sulem, and C. Mokbel, "Combining slanted-frame classifiers for improved HMM-based Arabic handwriting recognition," IEEE Transactions on Pattern Analysis and Machine Intelligence, vol. 31, pp. 1165-1177, 2009.

[5] A. Amin, "Off-line Arabic character recognition," Pattern Recognition, vol. 31, no. 5, pp. 517-530, 1998.

[6] N. A. Shaikh and Z. A. Shaikh, "A generalized thinning algorithm for cursive and non-cursive language scripts," in Proceedings of the 2005 Pakistan Section Multitopic Conference, pp. 1-4, Karachi, Pakistan, December 2005.

[7] P. Dhande and R. Kharat, "Recognition of cursive english handwritten characters," in Proceedings of the 2017 International Conference on Trends in Electronics and Informatics (ICEI), pp. 199-203, Tirunelveli, India, May 2017.

[8] X. Qin, J. Jiang, W. Fan, and C. Yuan, "Chinese cursive character detection method," The Journal of Engineering, vol. 2020, no. 13, pp. 626-629, 2020.

[9] K. Ueki, T. Kojima, R. Mutou, R. S. Nezhad, and Y. Hagiwara, "Recognition of Japanese connected cursive characters using multiple softmax outputs," in Proceedings of the 2020 IEEE Conference on Multimedia Information Processing and Retrieval (MIPR), pp. 127-130, Guangdong, China, August 2020.

[10] H. Ke and I. K. Sethi, "Off-line cursive handwriting segmentation," in Proceedings of 3rd International Conference on Document Analysis and Recognition, pp. 894-897, Montral, Canada, August 1995. 
[11] J. H. Kim and J. J. Lee, “A unified network-based approach for recognition of cursive handwritings in mixed languages: a case study on Hangul and Roman mixture," in Proceedings of the IEEE Colloquium on Handwriting and Pen-Based Input, pp. 6/1-6/4, London, UK,, March 1994.

[12] J. Sternby and C. Friberg, "The recognition graph-language independent adaptable on-line cursive script recognition," in Proceedings of the Eighth International Conference on Document Analysis and Recognition (ICDAR'05), pp. 14-18, Seoul,, South Korea, August 2005.

[13] R. Ahmad, M. Z. Afzal, S. F. Rashid, M. Liwicki, A. Dengel, and T. Breuel, "Recognizable units in Pashto language for OCR," in Proceedings of the 2015 13th International Conference on Document Analysis and Recognition (ICDAR), pp. 1246-1250, Tunis, Tunisia, August 2015.

[14] N. Hassan, "Recognition of Arabic cursive handwriting," in Proceedings of the Geometric Modeling and Imaging--New Trends (GMAI'06), pp. 135-140, London, UK, July 2006.

[15] M. R. Hashemi, O. Fatemi, and R. Safavi, "Persian cursive script recognition," in Proceedings of the 3rd International Conference on Document Analysis and Recognition, pp. 869873, Montreal, Canada, August 1995.

[16] T. Erdogan and O. Erdogan, "An analysis of the legibility of cursive handwriting of prospective primary school teachers," Procedia - Social and Behavioral Sciences, vol. 46, pp. 52145218, 2012.

[17] O. Samanta, A. Roy, S. K. Parui, and U. Bhattacharya, "An HMM framework based on spherical-linear features for online cursive handwriting recognition," Information Sciences, vol. 441, pp. 133-151, 2018.

[18] F. Camastra, "A SVM-based cursive character recognizer," Pattern Recognition, vol. 40, no. 12, pp. 3721-3727, 2007.

[19] S. M. Darwish and H. M. ELgohary, Building an Expert System for Printer Forensics: A New Printer Identification Model Based on Niching Genetic Algorithm, Wiley, Hoboken, NJ, USA, 2020.

[20] W.-T. Chen and T.-R. Chou, "A hierarchical deformation model for on-line cursive script recognition," Pattern Recognition, vol. 27, no. 2, pp. 205-219, 1994.

[21] H. Lee and B. Verma, "Binary segmentation algorithm for english cursive handwriting recognition," Pattern Recognition, vol. 45, no. 4, pp. 1306-1317, 2012.

[22] T. S. El-Sheikh and R. M. Guindi, "Computer recognition of Arabic cursive scripts," Pattern Recognition, vol. 21, no. 4, pp. 293-302, 1988.

[23] A. K. Bhunia, P. P. Roy, A. Mohta, and U. Pal, "Cross-language framework for word recognition and spotting of Indic scripts," Pattern Recognition, vol. 79, pp. 12-31, 2018.

[24] A. A. Chandio, M. Asikuzzaman, M. Pickering, and M. Leghari, "Cursive-text: a comprehensive dataset for endto-end Urdu text recognition in natural scene images," Data in Brief, vol. 31, Article ID 105749, 2020.

[25] A. Aisyah, N. Hieda, M. Nezu, and N. Ibrahim, "Designing hiragana learning materials for Japanese language course in UKM," Procedia-Social and Behavioral Sciences, vol. 59, pp. 451-458, 2012.

[26] I. S. I. Abuhaiba, "Discrete script or cursive language identification from document images," Journal of King Saud University - Engineering Sciences, vol. 16, no. 2, pp. 253-268, 2004.

[27] J. Hellige and M. Adamson, "Hemispheric differences in processing handwritten cursives," Brain and Language, vol. 102, no. 3, pp. 215-227, 2007.
[28] A. Jalali and M. Lee, "High cursive traditional Asian character recognition using integrated adaptive constraints in ensemble of densenet and Inception models," Pattern Recognition Letters, vol. 131, pp. 172-177, 2020.

[29] W. Cho, S.-W. Lee, and J. H. Kim, "Modeling and recognition of cursive words with hidden Markov models," Pattern Recognition, vol. 28, no. 12, pp. 1941-1953, 1995.

[30] S. Naz, A. I. Umar, R. Ahmad et al., "Offline cursive UrduNastaliq script recognition using multidimensional recurrent neural networks," Neurocomputing, vol. 177, pp. 228-241, 2016.

[31] M. Schambach, "Recurrent HMMs and cursive handwriting recognition graphs," in Proceedings of the 2009 10th International Conference on Document Analysis and Recognition, pp. 1146-1150, Barcelona, Spain, July 2009.

[32] T. G. Rose and L. J. Evett, "Semantic analysis for large vocabulary cursive script recognition," in Proceedings of 2 nd International Conference on Document Analysis and Recognition (ICDAR, pp. 236-239, Tsukuba, Japan, October 1993.

[33] R. J. Kannan, R. Prabhakar, and R. M. Suresh, "Off-line cursive handwritten Tamil character recognition," in Proceedings of the 2008 International Conference on Security Technology, pp. 159-164, Hainan Island, China, December 2008.

[34] A. A. Chandio, M. Asikuzzaman, and M. R. Pickering, "Cursive character recognition in natural scene images using a multilevel convolutional neural network fusion," IEEE Access, vol. 8, pp. 109054-109070, 2020.

[35] J. Danna, D. Massendari, B. Furnari, and S. Ducrot, "The optimal viewing position effect in printed versus cursive words: evidence of a reading cost for the cursive font," Acta Psychologica, vol. 188, pp. 110-121, 2018.

[36] B. Verma and H. Lee, "Segment confidence-based binary segmentation (SCBS) for cursive handwritten words," Expert Systems with Applications, vol. 38, no. 9, pp. 11167-11175, 2011.

[37] J. H. AlKhateeb, J. Ren, J. Jiang, and H. Al-Muhtaseb, “Offline handwritten Arabic cursive text recognition using Hidden Markov Models and re-ranking," Pattern Recognition Letters, vol. 32, no. 8, pp. 1081-1088, 2011.

[38] T. Abu-Ain, S. N. H. S. Abdullah, B. Bataineh, W. Abu-Ain, and K. Omar, "Text normalization framework for handwritten cursive languages by detection and straightness the writing baseline," Procedia Technology, vol. 11, pp. 666-671, 2013.

[39] R. Mouhcine, A. Mustapha, and M. Zouhir, "Recognition of cursive Arabic handwritten text using embedded training based on HMMs," Journal of Electrical Systems and Information Technology, vol. 5, no. 2, pp. 245-251, 2018.

[40] K. Manjusha, M. A. Kumar, and K. P. Soman, "On developing handwritten character image database for Malayalam language script," Engineering Science and Technology, an International Journal, vol. 22, no. 2, pp. 637-645, 2019.

[41] S. Naz, K. Hayat, M. Imran Razzak, M. Waqas Anwar, S. A. Madani, and S. U. Khan, "The optical character recognition of Urdu-like cursive scripts," Pattern Recognition, vol. 47, no. 3, pp. 1229-1248, 2014. 